\title{
Alcances y límites de la gobernanza urbana: incentivos públicos $y$ estrategias empresariales en los distritos de la ciudad de Buenos Aires
}

Carolina Gonzalez-Redondo. Universidad de Buenos Aires, Buenos Aires, Argentina.

RESUMEN | El artículo reflexiona sobre los procesos de articulación público-privada que se despliegan en el marco de la política de distritos en el sur de la ciudad de Buenos Aires (2008-2019). Particularmente se pregunta: ¿cuáles son las respuestas que producen los actores empresariales ante los distintos incentivos públicos?, ¿qué estrategias y vínculos se privilegian en cada distrito?, ¿qué procesos y dinámicas se propician en el territorio? Para ello, desde un abordaje cualitativo y recuperando el andamiaje teórico de la sociología económica, se estudian las estrategias y percepciones de los actores empresariales involucrados en dicha política. Los hallazgos permiten cuestionar los principales supuestos en que se basa esta política y, a la vez, reflexionar sobre los alcances y limitaciones de los procesos de gobernanza urbana.

PALABRAS CLAVE | competitividad urbana, gobierno local, política urbana.

ABSTRACT | The article reflects on the public-private articulation processes that take place within the framework of the district policy in the southern Buenos Aires (2008-2019). In particular, the article questions the responses of corporate actors to public incentives regarding the type of strategies and relationships takes place in each district, and the kind of processes and dynamics that are promoted in the territory. From a qualitative approach, and recovering the theoretical perspective of economic sociology, the paper studies the strategies and perceptions of the corporate actors involved in this policy. The findings allow us to question the main assumptions on which this policy is based and to reflect on the scope and limits to urban governances processes.

KEYWORDS | urban competitiveness, local government, urban policy.

Recibido el 30 de julio de 2020, aprobado el 26 de octubre de 2020.

E-mail: carolina.gredondo@gmail.com 


\section{Introducción}

Desde 2008, el Gobierno de la Ciudad de Buenos Aires (GCBA) crea distritos económicos en áreas donde se promueve la radicación de empresas de un mismo sector económico. Estos distritos conforman una política paraguas que profundiza el proceso de renovación urbana desplegado en el sur de la ciudad desde la década de los noventa. En sintonía con los discursos sobre gobernanza urbana (Banco Mundial [вм], 2009; Comisión Económica para América Latina y el Caribe [CEPAL], 2018; Naciones Unidas [ONU], 2017), y retomando premisas del modelo de clúster (Porter, 1995), esta política se apoya en la "sinergia público-privada" para desarrollar estas "zonas deterioradas". Así, el gobierno local produce intervenciones tendientes a crear el "clima de negocios" necesario para la atracción de estos emprendimientos. Entre ellas, se destacan los incentivos tributarios, financieros e inmobiliarios; las estrategias de articulación con actores empresariales; y la inversión pública orientada a la renovación urbana (Gonzalez Redondo, 2020b). A pesar de estos esfuerzos públicos, la respuesta de los actores empresariales es heterogénea, acompańando con distinta intensidad y ritmos las intervenciones desplegadas en el marco de esta política. Este artículo indaga en las estrategias y percepciones de los actores empresariales y agentes culturales involucrados en dicha política. Para ello, nos preguntamos ¿cuáles son las respuestas que producen estos actores ante los incentivos públicos?, ¿qué estrategias y vínculos se privilegian en cada distrito?, ¿qué procesos se observan en el territorio?

En este artículo, focalizamos en tres de los cinco distritos económicos: Artes, Diseńo y Tecnológico (Figura 1). En el Distrito Tecnológico (DT), de 2008, se promueve la radicación de empresas vinculadas a las Tecnologías de la Información y la Comunicación (TIC) en un polígono que abarca Parque Patricios, y parte de Nueva Pompeya y Boedo. A fines de 2012, se creó el Distrito de las Artes (DA), que busca la instalación de emprendimientos culturales en La Boca y el sector sur de San Telmo. En el Distrito del Diseńo (DDi), creado en 2013 en Barracas, se incentiva la instalación de firmas dedicadas al diseño en distintos rubros. El Distrito Audiovisual fue excluido por situarse en el norte de la ciudad, con características socioterritoriales muy diferentes. El Distrito del Deporte se excluyó de este trabajo, porque allí la intervención estatal cobra características que lo diferencian de nuestros casos de estudio (Arqueros, 2017).

Nos apoyamos en una estrategia cualitativa, con orientación empírica e interpretativa. Combinamos diversas fuentes y técnicas, como documentos vinculados a la política (marcos normativos, gacetillas de prensa y materiales gráficos); y entrevistas semiestructuradas a actores involucrados en la implementación de los distritos (empresarios, agentes culturales, funcionarios del GCBA). Para su abordaje, combinamos el análisis interpretativo con el trabajo con sistemas de información geográfica (sIG) y con un dispositivo para el análisis de los discursos. Recuperamos información elaborada por organismos del GCBA (inversiones en obras públicas, información sobre transporte, infraestructura y equipamiento urbano y localización de beneficiarios de 
los distritos ${ }^{1}$ y la procesamos mediante sIG. El análisis de información georreferenciada permite ver la distribución desigual de las inversiones públicas y privadas en los tres distritos. El análisis discusivo nos acerca a las percepciones de los actores empresariales y agentes culturales entrevistados. Construimos un dispositivo que, retomando la perspectiva teórica de la escuela francesa, combina elementos de las teorías de la enunciación y la argumentación (Gonzalez Redondo, 2020a).

\section{Figura I | Distritos económicos, Ciudad Autónoma de Buenos Aires (CABA)}

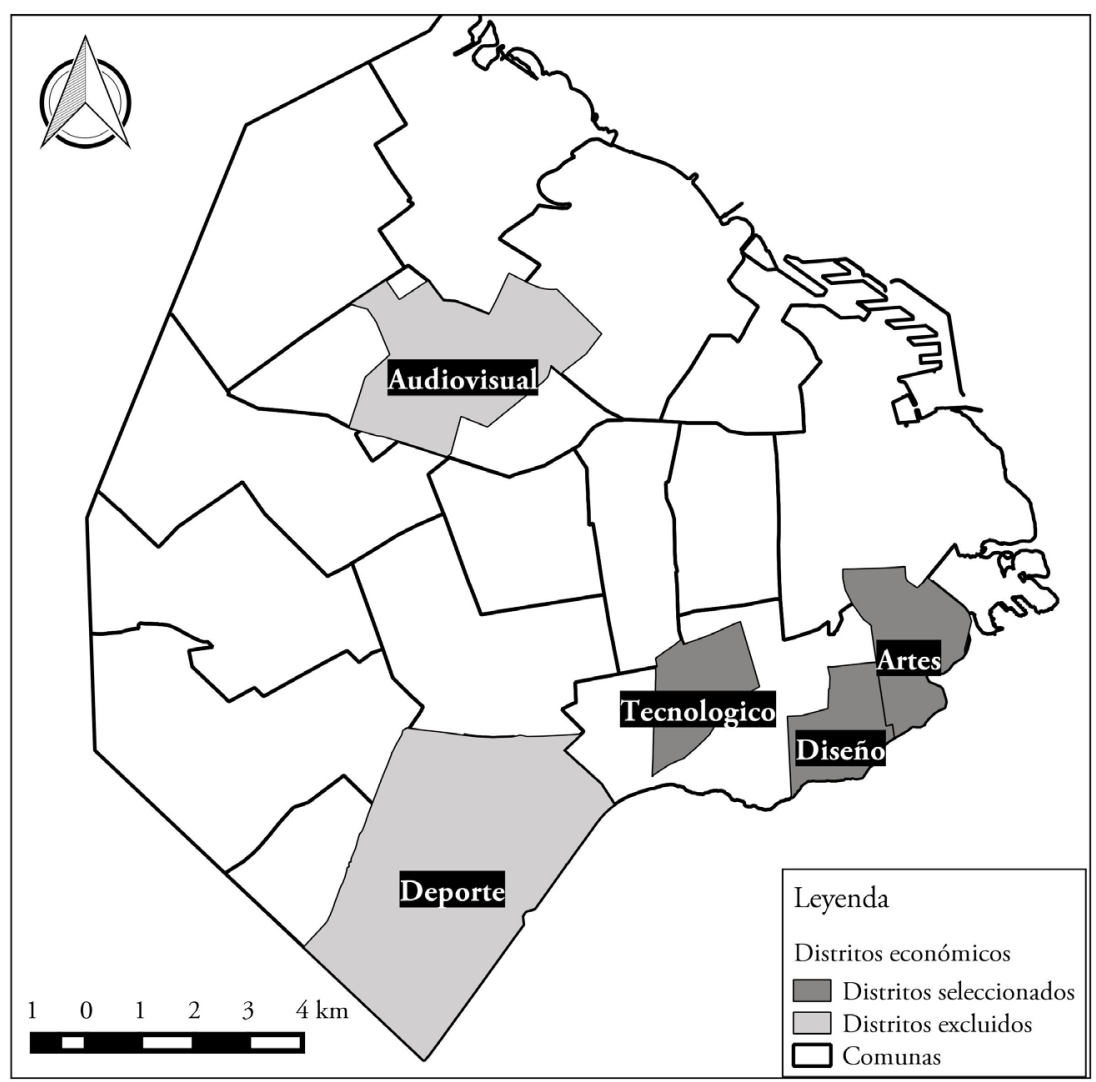

FUENTE: ELABORACIÓN PROPIA

El artículo produce aportes en dos sentidos. Primero, el análisis comparado de los distritos permite cuestionar sus modelos de referencia y los principales supuestos en que se basa esta política, produciendo un aporte a los estudios sobre movilidad de políticas (Peck \& Theodore, 2010). Segundo, introduce el andamiaje teórico de la sociología económica para analizar las relaciones entre actores estatales y empresariales en la producción de la ciudad. Este abordaje echa luz sobre los procesos de 
articulación público-privada, invitándonos a reflexionar sobre los alcances y limitaciones de la gobernanza urbana.

\section{Antecedentes}

Las características que asume la política de distritos son producto de la interacción entre procesos de acumulación de capital a escala global y las características socioterritoriales, económicas y políticas del escenario nacional y local. Al retomar muchas de las premisas de los modelos sobre desarrollo territorial que se implementan en otras latitudes, la política de distritos se vincula con procesos de escala global. Pero, a la vez, al interactuar con el territorio, esta política adopta características propias del ámbito local, produciendo modalidades específicas de neoliberalización (Harvey, 2005; Peet, 2001; Theodore et al., 2009).

Atendiendo a la escala global, en trabajos previos (Gonzalez Redondo, 2018, 2020c) advertimos que los distritos recuperan elementos de un modelo de gestión urbana de gran difusión, como es el clúster (Porter, 1995, 1998). El supuesto central de la noción de clúster es que la proximidad geográfica entre empresas e instituciones de un mismo sector económico genera intercambios que favorecen el crecimiento de toda la cadena, a través de las denominadas "sinergias". Una segunda premisa es que los clústeres motorizan procesos de desarrollo en la medida en que generan actividad económica, que derrama en inversiones y empleo; según este modelo, el Estado debe adoptar un rol de facilitador, potenciando las ventajas competitivas de su territorio que colaboran en la gestación del "clima de negocios" (Porter, 1995, 1998).

Estos supuestos del clúster se articulan conceptualmente con los enfoques sobre gobernanza que cobraron fuerza durante los noventa (Aguilar, 2006; Marques, 2013) y destacan la mayor injerencia de actores no gubernamentales en la definición e implementación de políticas públicas y en la prestación de servicios públicos. En su proceso de institucionalización y circulación, la noción de gobernanza se transformó en un concepto normativo que seńala el tipo de gobierno "apropiado" para el nuevo milenio (Aguilar, 2006; Marques, 2013). Es precisamente este concepto normativo de gobernanza el que tuvo mayor circulación en América Latina por parte de los organismos de cooperación internacional. En el marco de los procesos de neoliberalización de los ańos noventa, las recomendaciones de organismos internacionales resaltaron determinadas características del proceso de gobernabilidad (como transparencia, eficacia, estabilidad institucional, seguridad jurídica, participación ciudadana) para garantizar el funcionamiento de las fuerzas del mercado. A la vez, promovieron la mayor participación del sector privado y de la sociedad civil en áreas hasta entonces reservadas al sector público (Zurbriggen, 2011), articulándose de este modo con las teorías neoliberales de la época. Llevada al plano local, la gobernanza urbana establece las bases para el buen gobierno de las ciudades, en el marco de los nuevos desafíos impuestos por la economía global. Uno de sus argumentos principales es que la complejidad de los problemas urbanos actuales dificulta su abordaje por parte del gobierno local, haciendo imprescindible la vinculación con otros actores no gubernamentales (BM, 2009; CEPAL, 2018; ONU, 2017). 
Ahora bien, los actores locales inciden fuertemente en las características que asume esta política, por lo que, en este artículo, analizamos cómo se produce la articulación entre actores estatales y empresariales en la implementación de la misma. En trabajos previos (Gonzalez Redondo, 2019, 2020b), analizamos cómo el gobierno local recupera estas premisas y asume un rol de facilitador y mediador, que apunta a generar oportunidades de negocio para atraer inversores a las áreas promocionadas. En este artículo nos centramos en las respuestas que producen los actores empresariales a estos incentivos públicos. Para ello, retomamos la perspectiva de la sociología económica (Beltrán, 2007; Castellani, 2006; Castellani \& Gaggero, 2012; Gaggero, 2012), para la cual el empresariado no constituye un cuerpo homogéneo, sino que se caracteriza por sus heterogeneidades (Beltrán, 2007; Castellani, 2006). De ahí que analicemos los modos en que los empresarios de cada distrito se vinculan entre sí. Asimismo, planteamos que los actores empresariales organizan sus acciones no solo a partir de las interpretaciones que hacen sobre la intervención pública y sobre la coyuntura en que esta se inscribe (Beltrán, 2007; Gaggero, 2012), sino también a partir de la lectura que hacen del territorio en que la política se desenvuelve. Por ello, para estudiar las estrategias empresariales, recurrimos también al análisis de sus percepciones sobre la acción pública, sus coyunturas y los territorios en que se despliega.

\section{Resultados y discusión}

\section{El Distrito Tecnológico: una apuesta sobre seguro}

A diez años de su creación, el Distrito Tecnológico es presentado como una política "exitosa", debido a sus abultadas cifras en cuanto a la cantidad de empresas radicadas, inversión privada y metros cuadrados construidos. En efecto, en el Dт hay 263 empresas, de las cuales solo nueve preexisten a su creación. Las narrativas sobre el distrito, así como los discursos de los empresarios, suelen tematizar la decisión de inversión en el distrito en términos de una "apuesta" ante la "oportunidad" generada por la creación del DT. Ahora bien, ¿en qué consisten esa "apuesta” y esa "oportunidad"?

Todas las empresas consultadas señalan, como primer factor de radicación, la exención en ingresos brutos (II. Bв.), que es percibida como un beneficio de envergadura para su actividad. En efecto, las exenciones en II. BB. en el DT representan una gran proporción de la inversión pública destinada a la política de distritos (Gonzalez Redondo, 2019). Sin embargo, la decisión de instalación en el Distrito Tecnológico no fue inmediata. Las empresas consultadas tardaron entre tres y nueve años en mudarse a Parque Patricios y lo hicieron cuando consideraron que era el "momento oportuno". Así, vemos que la racionalidad económica -entendida como el comportamiento racional orientado por fines económicos- es una de las dimensiones que organiza la acción empresarial, pero no la única (Beltrán, 2007). En ella intervienen otros factores, como las interpretaciones que los empresarios hacen respecto del accionar público y de la coyuntura política, económica y social en que este se inscribe. De ahí que las decisiones empresariales se materialicen en el lugar y el momento "oportunos", siguiendo la temporalidad y la ubicación de las principales intervenciones públicas. De hecho, la mayor cantidad de empresas se 
radica una vez que se consolida la gestión del partido Propuesta Republicana (PRO) en el gobierno local (con la reelección de 2011), y se materializan dos de las grandes inversiones prometidas: la extensión de la línea $\mathrm{H}$ del subte (2013) y la radicación de la Jefatura de Gobierno (2015). Además, la mayoría de las empresas se instala en el sector más consolidado, hacia el norte del distrito, donde se concentran las principales intervenciones públicas.

FIgURA 2 | Amenidades e inversiones públicas y privadas, Distrito Tecnológico (DT)

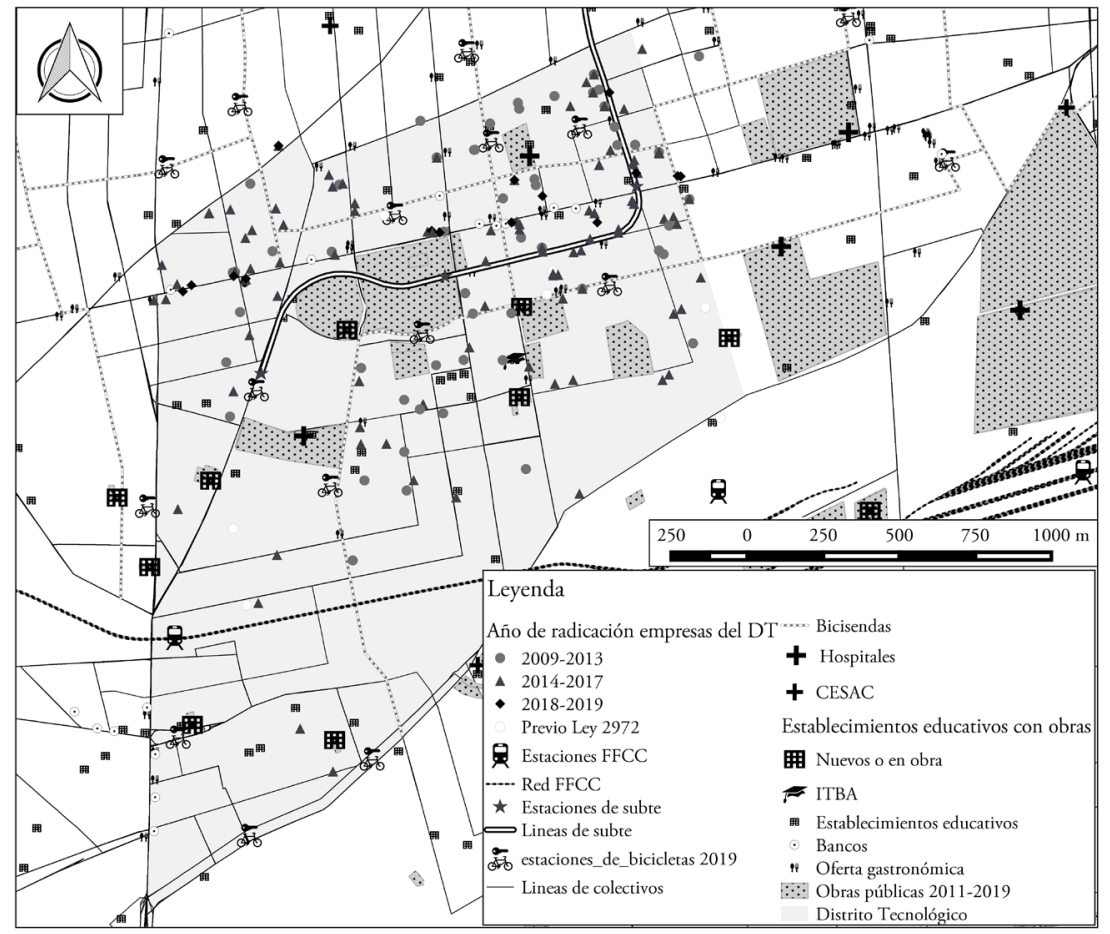

FUENTE: ELABORACIÓN PROPIA CON BASE EN INFORMACIÓN DEL GCBA

La "apuesta" se limita a realizar una inversión segura, aprovechando la "oportunidad" que suponen los incentivos públicos. El gobierno local garantiza a las empresas una rentabilidad extraordinaria, con las exenciones fiscales y los créditos blandos, a la vez que asegura las condiciones urbanas necesarias para ejercer su actividad y valorizar sus inversiones (Gonzalez Redondo, 2019, 2020b). Esto permite concebir el Distrito Tecnológico como un ámbito privilegiado de acumulación (Castellani, 2006), en el que tanto empresas TIC como del sector inmobiliario internalizan amplios márgenes de beneficios extraordinarios -entre ellos, los incrementos en la renta del suelo (Jaramillo, 2009) - a partir de la transferencia de aquellos recursos públicos que suponen los beneficios y las inversiones desplegadas por el gobierno local.

Asimismo, al no exigirse metas de cumplimiento ni contraprestaciones a cambio de estos beneficios, la reciprocidad (Castellani \& Borrastero, 2011) queda sujeta 
a la (buena) voluntad y predisposición de los actores empresariales, primando estrategias de vinculación y de inversión orientadas por la lógica de la ganancia. Así, las sinergias público-privadas y el denominado "efecto clúster" (Porter, 1998) se reducen a las iniciativas de aquellas empresas que buscan ser "algo más" que "ingreso-brutistas":

Me gustaría que nosotros no seamos un distrito de ingresos brutos, "los ingresobrutistas" nada más. (...). Igual estaría bueno que se quede, porque mirá lo que se hace con un $4 \%$ (...). Tener esta prebenda impositiva, cómo ha motivado. Yo no quisiera ser nada más que esto. Y creo que casi todos los [empresarios] que estamos acá no somos nada más que esto. No pusimos cuatro chapas. Y todos además tenemos más inversiones que esta; todos compramos más propiedades, tenemos más metros, hicimos más cosas que estas. (Entrevista a Mariana, empresaria del DT, marzo de 2019)

En esta secuencia, el enunciador se construye a sí mismo en oposición a la figura "ingreso-brutistas", una versión costumizada del estereotipo (Amossy, 2000) de "chori-planeros", referido en términos peyorativos a aquellos sujetos poseedores de planes sociales. Se trata de empresarios que no se limitan a recibir un beneficio fiscal, "una prebenda impositiva", sino que hacen "mucho más". Un "mucho más" asociado a una serie de inversiones inmobiliarias. Estas inversiones, que exceden la realización de las oportunidades que el distrito ofrece, se orientan a reforzar la valorización de sus propios emprendimientos. Esta tematización sobre las empresas que "se ponen la camiseta [del distrito]", "quieren dejar un legado a lo público" y "realmente están interesadas en el desarrollo de la zona", se asocia a aquellas firmas que están actualmente nucleadas bajo la figura del Board del Distrito Tecnológico, y se define por oposición al anterior Consorcio de Industrias del Distrito Tecnológico de la Ciudad de Buenos Aires, GigaBa.

Como anticipamos, uno de los supuestos centrales del modelo de clúster es que la concentración geográfica de empresas y agentes de un mismo sector económico genera dinámicas de cooperación y competencia que favorecen el crecimiento de toda la cadena (Porter, 1995, 1998). Con el fin de motorizar este "efecto clúster", el gobierno local promovió la creación de estas dos figuras, Gigaba y Board. Hasta el año 2016 funcionó el Consorcio, integrado por las empresas de mayor envergadura del distrito (Goicoechea, 2016), que rápidamente adquirió un formato de cámara, representando los intereses de sus asociados y formulándolos en términos de demandas hacia el Estado. Si bien la presión del Consorcio se tradujo en algunas acciones concretas, la heterogeneidad de los actores empresariales (Arqueros, 2017; Beltrán, 2007; Castellani \& Gaggero, 2012; Dossi \& Lissin, 2011) y sus intereses dificultaron la generación de consensos y obturaron la posibilidad de sostener en el tiempo esta acción empresarial organizada (Dossi \& Lissin, 2011), haciendo que el Consorcio perdiera peso, hasta dejar de funcionar.

Frente a este "organismo para hacer lobby político" al que "no le fue bien", el Board se presenta como un espacio de intercambio integrado por aquellas empresas que "quieren devolver el beneficio que tienen" y "hacer cosas por el distrito". En funcionamiento desde 2017, el Board está integrado por algunas empresas 
referentes del DT, representantes de universidades privadas, y funcionarios y agentes del gobierno local. Estos actores se reúnen mensualmente y ponen en común situaciones propias de la actividad y del distrito. Entre sus principales iniciativas, se destacan la estrategia de internacionalización del distrito y algunas acciones que implican una contraprestación al sector público, como la cesión de espacios dentro de las oficinas corporativas para que el GCBA realice sus programas de capacitación, y la colaboración para financiar eventos del distrito y misiones comerciales en el exterior. Más allá de estos intercambios puntuales, por el momento no parece haberse generado una dinámica de cooperación que redunde en procesos innovadores o en el crecimiento de la cadena de valor radicada en el distrito. Los primeros intentos de generar las sinergias del "efecto clúster" se vieron frustrados por la incapacidad del Consorcio para aglutinar los diversos intereses de sus miembros y redefinirlos en un interés común que se sostenga en el tiempo (Dossi \& Lissin, 2011). Si bien el Board pareciera encaminarse en esa dirección, todavía está "encontrando su lugar en el mundo". ${ }^{2}$

Otros vínculos más virtuosos parecen tejerse entre empresas TIC y agentes inmobiliarios con el fin de aprovechar la "oportunidad inmobiliaria" generada por esta política, a partir de una combinación de factores: la disponibilidad de terrenos e inmuebles adecuados para este tipo de actividad en una zona con una baja incidencia del suelo; la exención de II. вв., que permite a estas empresas redirigir estas abultadas sumas de dinero a inversiones inmobiliarias; el acceso a créditos a tasa subsidiada; todo ello fortalecido mediante inversión pública tendiente a reforzar los atributos de centralidad de la zona. Esto contribuyó para que la inversión inmobiliaria resultara una "oportunidad" muy conveniente para las empresas TIC, no solo por la inversión en sí, sino también por la posibilidad de diversificar su capital; por ejemplo, subalquilando una parte de sus edificios a otras empresas o invirtiendo en otros emprendimientos corporativos en asociación con desarrolladores del distrito. Así, las relaciones entre desarrolladores y empresas Tic radicadas en el distrito parecen ser virtuosas: mientras los promotores aportan su expertise en lo que respecta a la gestión del suelo, el conocimiento de la norma urbana, el diseño del proyecto y la dirección de obra, las empresas garantizan el financiamiento -un elemento clave en contextos de restricciones financieras como el presente- y mejoran la rentabilidad del proyecto al aportar las exenciones tributarias asociadas a la actividad inmobiliaria.

Así, se generó una demanda de oficinas que, a su vez, supuso una "oportunidad" para los desarrolladores inmobiliarios, a quienes se les garantizó uno de sus insumos fundamentales: el financiamiento:

En la industria del corporativo, en general las empresas no compran, las empresas alquilan. Entonces, cuando uno quiere hacer edificios de oficinas, tiene que buscar o con capital propio, o inversores, o deuda, la forma de poder terminar el edificio para después poder alquilarlo, porque el cliente final alquila. La particularidad que tuvo el distrito, y nos permitió mucho, es que como el mejor aprovechamiento del

2 Entrevista al director general de Finanzas e Infraestructura del Instituto Tecnológico de Buenos Aires (гтвA), mayo de 2019. 
beneficio fiscal provenía de la compra y no del alquiler, las empresas compraban. Entonces uno podía apalancar proyectos con el cliente final (...). La parte comercial era más fácil, no tenías que ir a convencer a un inversor que invierta en Parque Patricios cuando nadie quería invertir en Parque Patricios, simplemente le decías a la empresa "te vas a ahorrar un montón de guita". (Entrevista a desarrollador inmobiliario "A" del DT, febrero de 2019)

De este modo, el acceso en propiedad por parte de las empresas Tic garantizó el capital que requiere el promotor para desarrollar la obra en un contexto de restricciones en el acceso al mercado financiero (Socoloff, 2018). Se conformó un mercado de oficinas, hasta entonces inexistente en esta zona, que inicialmente consistió en algunas operaciones de refuncionalización de galpones y la construcción de emprendimientos de menor categoría que, siguiendo la demanda de las empresas, se fue complejizando y ampliando su oferta hacia edificios de mayor envergadura, tanto en tamaño como en calidad (Goicoechea, 2016). También la localización de las inversiones inmobiliarias acompañó la demanda de las empresas, generándose una distribución desigual que, básicamente, coincide con la ubicación de las inversiones públicas (Figura 2).

Esta oferta progresiva y variada de edificios de oficinas fue a la vez causa y efecto de las estrategias de los agentes inmobiliarios, quienes supieron aprovechar la "oportunidad" generada a raíz de la creación del distrito, haciendo espacio para la intervención de una gama muy heterogénea de desarrolladores. En este caso sí parece haber operado un equilibrio delicado entre dinámicas de cooperación y de competencia, uno de los supuestos del clúster:

(...) los desarrolladores, que tenemos grupo de WhatsApp, tenemos todos re buena onda; creo que también supimos ser inteligentes de no pisarnos. De ir generando productos que no se pisaban. En general, a la final nunca llegábamos más de dos para un perfil de empresa. (Entrevista a desarrollador inmobiliario "A" del DT, febrero de 2019)

El mayor dinamismo del mercado inmobiliario en la zona del DT se manifestó, a su vez, en la valorización del suelo, tanto en lo que respecta a terrenos como al mercado de oficinas (Lerena, 2019; Lerena \& Gonzalez Redondo, 2019). De este modo, las intervenciones públicas orientadas a modificar la estructura de usos del suelo de la zona, junto con las inversiones destinadas a mejorar sus atributos de centralidad, modificaron las rentas secundarias potenciales, impactando en la estructura de rentas territoriales (Jaramillo, 2009). De todas formas, la valorización inmobiliaria no supuso un obstáculo para la localización de empresas, dado que estos aumentos se compensaron con los incentivos fiscales. En este sentido, el esfuerzo público se orientó a garantizar la rentabilidad del sector privado e hizo que, aun pasando los años, invertir en el Distrito Tecnológico continuara siendo una "oportunidad".

\section{Distrito de las Artes: la apuesta pendiente}

A diferencia de las representaciones en torno a su modelo (el DT), el Distrito de las Artes es percibido, por los actores privados entrevistados como un "fracaso", que se atribuye al hecho de haber copiado un modelo que no se ajusta ni a las 
características del territorio, ni de los actores, ni de las actividades promovidas. En el DA, 147 agentes radicados expresan una gran heterogeneidad en cuanto a las actividades que realizan, el tamańo del proyecto y el tiempo que llevan en el barrio. A su vez, de estos 147 agentes, solo 66 (un 45\%) perciben las exenciones impositivas. Son cifras que se explican por la poca atractividad del beneficio, dado que la actividad artística está exenta de ingresos brutos en toda la ciudad.

Reconociendo de antemano la poca atractividad de los beneficios impositivos, en la ley del DA se incluyó la figura del desarrollador de infraestructura artística como un modo de incentivar a los inversores "artísticos". Sin embargo, estos ajustes al modelo no garantizaron la radicación de emprendimientos en el distrito, dado que los incentivos para estos actores tampoco resultaron tan atractivos. De hecho, los tres desarrolladores de infraestructura entrevistados consideran que el pago a cuenta de II. Bв., equivalente al 25\% de la inversión realizada, "no sirve de nada", ${ }^{3}$ debido a los plazos que rigen para el recupero.

Ante las valoraciones negativas sobre los incentivos, cabe preguntarse por qué causa estos actores comienzan a desarrollar actividades artísticas en el distrito. Aquí las decisiones de radicación en el lugar incluyen una diversidad de factores que dan cuenta de las múltiples dimensiones que orientan la acción empresarial (Beltrán, 2007). Entre ellos, destacan algunos atributos del barrio La Boca, como su tradición artística, su pasado migrante y su cercanía con el microcentro porteńo. Sin embargo, en casi todos los casos, la radicación en el distrito se presenta en términos de una "apuesta". Ahora bien, los motivos y efectos de esta "apuesta" son bien diferentes a los que identificamos en el Distrito Tecnológico.

Dos de los emprendimientos nuevos fueron desarrollados por empresas que ya tenían propiedades en el barrio La Boca. En uno de los casos es explícito que el motivo de radicación constituye una "apuesta" por la valorización inmobiliaria de la zona, lo que representaría una valorización de las distintas propiedades que la empresa tiene en el distrito. En otro caso, la "apuesta” por la valorización se presenta mediante eufemismos como "un fanatismo por La Boca" o "volver a poner en valor estético la zona circundante". " Esta empresa, con una larga tradición en el barrio, tiene "la mayor cantidad de propiedades de La Boca", según otro inversor de la zona. Así, mientras que en el DT la "apuesta" fue más bien una inversión sobre seguro en el marco de una "oportunidad" propiciada por la creación del distrito, en el DA la "apuesta" constituye una estrategia de especulación inductiva (Jaramillo, 2009), con la confianza en que "tarde o temprano" terminará siendo una inversión rentable, precisamente debido a las condiciones territoriales de la zona:

La Boca es, y te lo sigue permitiendo, la zona de menor valor de metro cuadrado. O sea, si uno quiere hacer una inversión de tierra fuerte, La Boca o Lugano, si querés, son los dos barrios más baratos para comprar tierra. Y si uno viaja a cualquier lado del mundo, lo primero que hace es buscar dónde hay un canal o dónde hay un río y caminar la costa. Son lugares que se terminan desarrollando, se desarrollan. artística en el DA, mayo de 2019.

4 Entrevista a Mario, encargado de un desarrollo de infraestructura artística en el DA, marzo de 2019. 
(Entrevista a Darío, director de una residencia de artistas y sala de exposición en el DA, marzo de 2019)

Así, las estrategias desplegadas por los actores empresariales -en este caso, del sector inmobiliario- no solo son moldeadas por sus interpretaciones de la coyuntura (Arqueros, 2017; Beltrán, 2007, 2011; Gaggero, 2012), sino también por sus lecturas sobre el territorio. Anticipar la localización de los potenciales demandantes del espacio construido es una de las tareas fundamentales de los promotores inmobiliarios (Socoloff, 2013, p. 59). De esta forma, mientras que Parque Patricios y Pompeya no estaban en el radar de los actores empresariales ni estatales, por lo que la creación del DT fue una sorpresa que generó rápidamente una "oportunidad" de inversión, el proceso de renovación urbana en La Boca desde los años noventa (Herzer, 2008; Rodríguez \& Di Virgilio, 2014) habilitó que distintos inversores fueran acumulando propiedades a lo largo del tiempo, esperando el momento adecuado para la captura de plusvalía.

Sin embargo, a diferencia de lo que sucedió en el DT, para quienes "apostaron" al Distrito de las Artes como un modo de valorizar sus inversiones, por el momento la "apuesta" no ha tenido éxito. Si bien se generaron algunos desarrollos inmobiliarios y hay otros en carpeta (Figura 3), en el Distrito de las Artes el "boom inmobiliario" todavía no ha explotado. Se observa una mayor cantidad de ofertas de terrenos, departamentos y locales comerciales que en el resto de los distritos, pero la mayoría se ubica en el sector más consolidado del DA, en el barrio de San Telmo (Lerena $\&$ Gonzalez Redondo, 2019). Lo mismo sucede con la dinámica de precios. Si bien se advierten incrementos en los precios promedio en dólares por metro cuadrado en terrenos, departamentos y locales, a juzgar por la localización de las ofertas, tales incrementos responden más a la influencia del área central y patrimonial, a la dinámica turística y a la valorización promedio de los ejes más consolidados del barrio, que a los efectos derivados de la creación del distrito (Lerena \& Gonzalez Redondo, 2019).

Para los desarrolladores inmobiliarios - muchos de los cuales son propietarios de tierra- todavía no es negocio construir en La Boca, debido a que no está garantizada una demanda que pague los precios del mercado. Según los actores entrevistados, son múltiples los factores que obturaron la realización de la "apuesta". Algunos se vinculan con las características de la ley y sus incentivos, otros con las particularidades de la actividad artística y otros con los condicionantes del territorio. Respecto a esto último, La Boca presenta los peores indicadores en términos sociohabitacionales, con altos índices de informalidad en la tenencia del suelo (Guevara, 2010). Estas cuestiones, sumadas a las disputas de las organizaciones sociales del barrio y las representaciones sobre la inseguridad en relación con La Boca, son sin duda factores que inciden en el devenir del Distrito de las Artes.

Ante esta situación, las iniciativas públicas y privadas han tendido -con sus limitaciones- a generar las condiciones propicias para la realización de la "apuesta". Esto se expresa, por un lado, en las inversiones desplegadas en el territorio (Figura 3) y, por el otro, en una estrategia de "dejar hacer" que habilita que el mercado se ocupe de "resolver" la problemática habitacional. De un lado, las dinámicas especulativas 
hacen que se multipliquen los procesos de desalojo en inmuebles de dominio privado. Del otro, una acción por omisión por parte del Estado que, al no atender la emergencia habitacional ni implementar acciones concretas para detener los desalojos, habilita procesos expulsivos por parte del mercado. Según relevamientos del Ministerio Público de la Defensa (MPD) junto con el Grupo de Vivienda de la organización La Boca Resiste y Propone, entre los años 2017 y 2018 se iniciaron 200 procesos de desalojo que involucran un total de 2.500 personas. Los desalojos se concentran en los sectores con mayor intervención pública y privada, en los alrededores de Caminito, la cancha de Boca, la calle Necochea y la Usina del Arte (Figura 3).

FIGURA 3 | Amenidades, inversiones públicas y privadas y desalojos, Distrito de las Artes

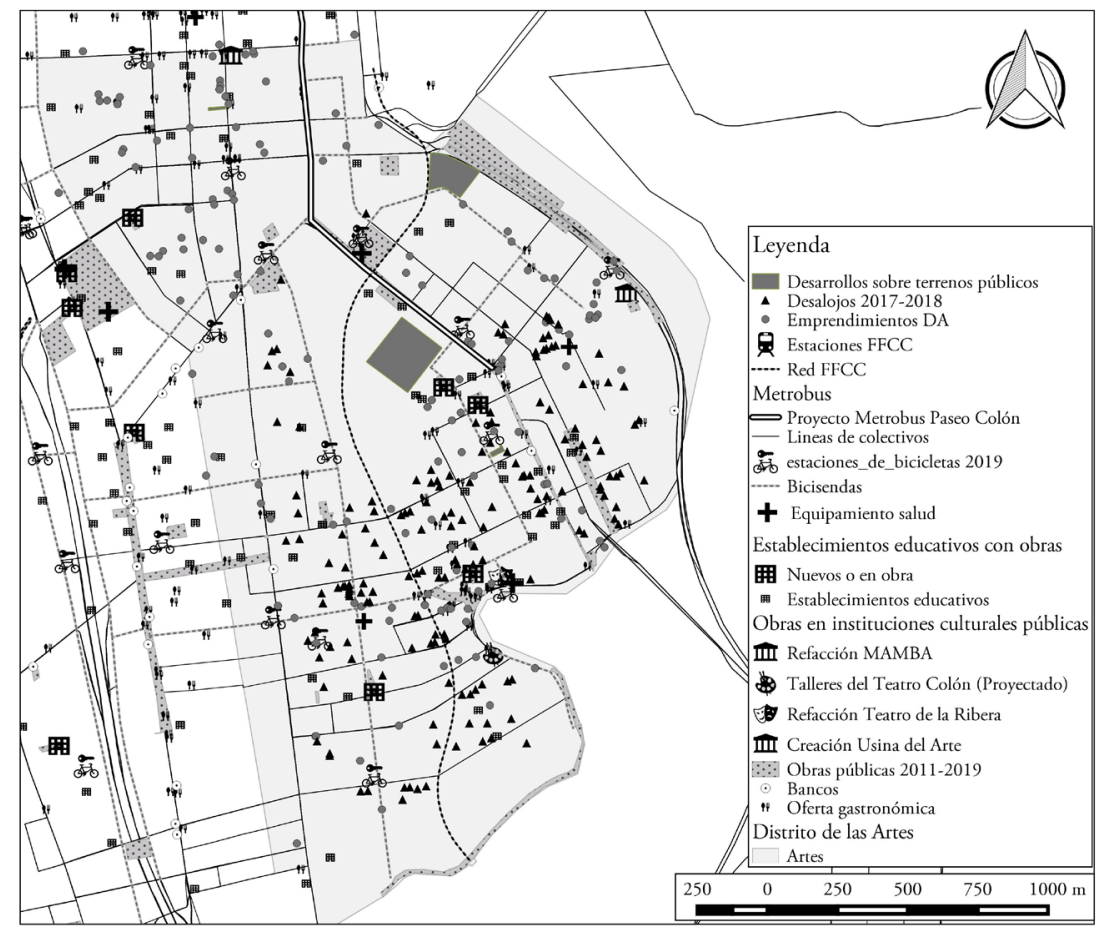

FUENTE: ELABORACIÓN PROPIA CON BASE EN INFORMACIÓN DEL GCBA. LA INFORMACIÓN SOBRE DESALOJOS FUE PROVISTA POR LA BOCA RESISTE Y PROPONE, Y EL MINISTERIO PÚBLICO DE LA DEFENSA (MPD)

Así, aun cuando todavía no se haya realizado la "apuesta" ni haya explotado el "boom inmobiliario", las intervenciones públicas y privadas ya están produciendo un impacto en el territorio, acentuando las desigualdades sociales y urbanas del área y afectando especialmente a los sectores más vulnerables. De todos modos, esta articulación público-privada no es implacable ni se produce sin contradicciones. Por lo 
contrario, las estrategias públicas y privadas son continuamente negociadas con las organizaciones locales e, incluso, con otros organismos del Estado.

Al igual que en el DT, a fines de 2016 el gobierno local incentivó la creación del Consorcio del Distrito de las Artes, como una iniciativa para vincular entre sí a los actores del distrito y propiciar de este modo el "efecto clúster". Sin embargo, la lista de integrantes del Consorcio (principalmente desarrolladores y agentes inmobiliarios, empresarios y propietarios de terrenos en La Boca, y unos pocos espacios, galerías y fundaciones de arte, en su mayoría nuevos en la zona), más los relatos de algunos de ellos, refieren más bien a un intento por conectar potenciales inversores con propietarios de tierra a fin de agilizar la realización de la "apuesta". Los entrevistados coinciden en que el Consorcio no prosperó, aunque difieren en las causas de su fracaso. Para algunos, el problema fue la heterogeneidad de los actores involucrados, con sus diferentes intereses y su falta de foco en el arte. Para otros, esto se combinó con el escaso acompańamiento por parte del gobierno local. Otros, en tanto, entendieron que el problema fue su acento demasiado crítico y poco propositivo. La diversidad de intereses, experiencias y lecturas sobre la política dificultaron -al igual que en el DT- el sostenimiento de una acción empresarial organizada (Dossi \& Lissin, 2011).

La heterogeneidad de los actores involucrados en el DA pareciera dar lugar a relaciones que corren por múltiples circuitos separados. Por un lado, los actuales miembros del Consorcio, que buscan consolidarse como un interlocutor con más peso para negociar sus necesidades con el sector público. Muchos de estos actores sostienen una estrategia especulativa, a la espera de que su "apuesta" finalmente se concrete. Otro circuito parece girar en torno a los espacios de artes visuales de obra contemporánea, los "recién llegados", que estrechan lazos para hacer exposiciones conjuntas. Luego, en otro circuito identificamos algunos agentes culturales con mayor antigüedad en el barrio que se articulan para proyectos puntuales. Por último, advertimos lazos más informales entre artistas del barrio que, por sus trayectorias e intereses, generan algunos trabajos en conjunto. En estos dos últimos casos, se trata de redes que preexisten al DA, cuya identidad no está dada por el distrito, sino por su pertenencia al barrio La Boca.

En el marco de estos circuitos aislados, conformados por actores muy disímiles entre sí, se insertan los esfuerzos públicos orientados a crear una identidad común bajo el paraguas del Distrito de las Artes. Así, surgen iniciativas que buscan congregar a (casi) todos los agentes culturales del distrito en un mismo circuito artístico, intentos que se ven frustrados por la negativa a participar por parte de algunos actores, o porque son considerados insuficientes por quienes sí participan. Ante este tipo de propuestas que buscan imponer una identidad común suprimiendo las diferencias, cabe recuperar el testimonio de uno de los espacios con mayor tradición en el barrio:

[Nos dicen:] "Che, articulemos, hagamos algo". No sé qué podemos hacer, ellos hacen arte contemporáneo y nosotros arte popular. Tenemos nuestro lugar, no sé qué podemos hacer (...). Después, el año pasado inventó el Ministerio de Cultura otra copia de las Ciudades Creativas, que los llamaron Barrios Creativos (...). Te 
obligaban a articular entre todos en un proyecto común. Yo tengo buena onda, pero no sé si puedo hacer un proyecto. ¿Por qué me obligás a que me junte? (...). No está ni bien ni mal, estamos en el mismo barrio, somos diversos, no tenemos por qué articular y trabajar juntos. (Entrevista a Gabriel, referente de un espacio cultural histórico del barrio La Boca, mayo de 2019)

Las dificultades para integrar a los distintos actores, con sus diversas trayectorias, intereses y expectativas, bajo una identidad propia del Distrito de las Artes invitan a reflexionar sobre los límites de estos modelos de clúster y los procesos de gobernanza. Ya vimos en el caso del Distrito Tecnológico cómo las relaciones entre los distintos actores son complejas y no necesariamente redundan en las dinámicas de aprendizaje y cooperación que se asumen en la base de los modelos. Estas dificultades se acentúan aún más en el Distrito de las Artes, debido a la heterogeneidad de los actores involucrados.

\section{Distrito del Diseño: del potencial a la supervivencia}

El Distrito del Diseño (DDi) tiene la menor cantidad de empresas radicadas (49 en total), de las cuales un 45\% (22) preexiste a su creación. Al igual que en el Distrito de las Artes, los potenciales beneficiarios del DDi son muy heterogéneos en relación con su actividad, dado que la ley está destinada a empresas que desarrollen, produzcan y/o comercialicen servicios o productos con diseńo en catorce rubros. Dada la preexistencia de muchas de estas empresas, las firmas se distribuyen en forma "salpicada" en todo el polígono del distrito, en lo que el propio gobierno local denomina como las tres "zonas de oportunidad" (Figura 4).

Los cuatro beneficiarios entrevistados expresan la heterogeneidad de las empresas radicadas en el distrito. Entrevistamos a un diseńador gráfico, que reside y trabaja en su hogar ubicado en el barrio Barracas; y a directivos o propietarios de tres empresas nacionales de distintos rubros (gráfica, muebles y alfombras), que realizan su actividad de diseño en Barracas con anterioridad a la creación del distrito. Entre los factores de radicación en la zona destacan la proximidad con el centro porteńo y el hecho de que sea un área con tradición industrial, lo que implica disponibilidad de espacios adecuados para estas actividades y la existencia de una zonificación que permite el uso industrial del suelo, todo en una ubicación privilegiada de la ciudad: "es como un lujo tener una industria dentro de la ciudad de Buenos Aires y en una ubicación a diez minutos del centro". 5

Otro empresario agrega, entre los factores de localización, el potencial de crecimiento y de valorización inmobiliaria. Así es como en el año 2008, cuando se vieron obligados a dejar sus instalaciones en el Partido de Avellaneda, decidieron mudarse a esta zona de la ciudad:

Obviamente que la diferencia de precios entre Avellaneda y Buenos Aires era muchísima (...). Esto era una inversión a futuro. Pensábamos que Buenos Aires tenía mucho crecimiento, más en esta zona que era una zona que le faltaba crecer (...). No imaginábamos que se iba a hacer un distrito, pero en todas las ciudades del 
mundo sucede que estas zonas medio relegadas, que fueron de producción, empiezan a tener un auge en algún momento, porque están muy desvalorizadas respecto a otras partes de la ciudad; entonces, como que las propiedades son más económicas. $\mathrm{Y}$ en algún momento a alguien se le ocurre ponerlas en valor, porque son como los últimos pulmones que tiene la ciudad, y entonces se revaloriza todo. Y es lo que nos sucedió a nosotros. (Entrevista a Esteban, empresario DDi, mayo de 2019)

FIGURA 4 | Amenidades, inversiones públicas y privadas y "zonas de oportunidad", Distrito del Diseño (DDi)

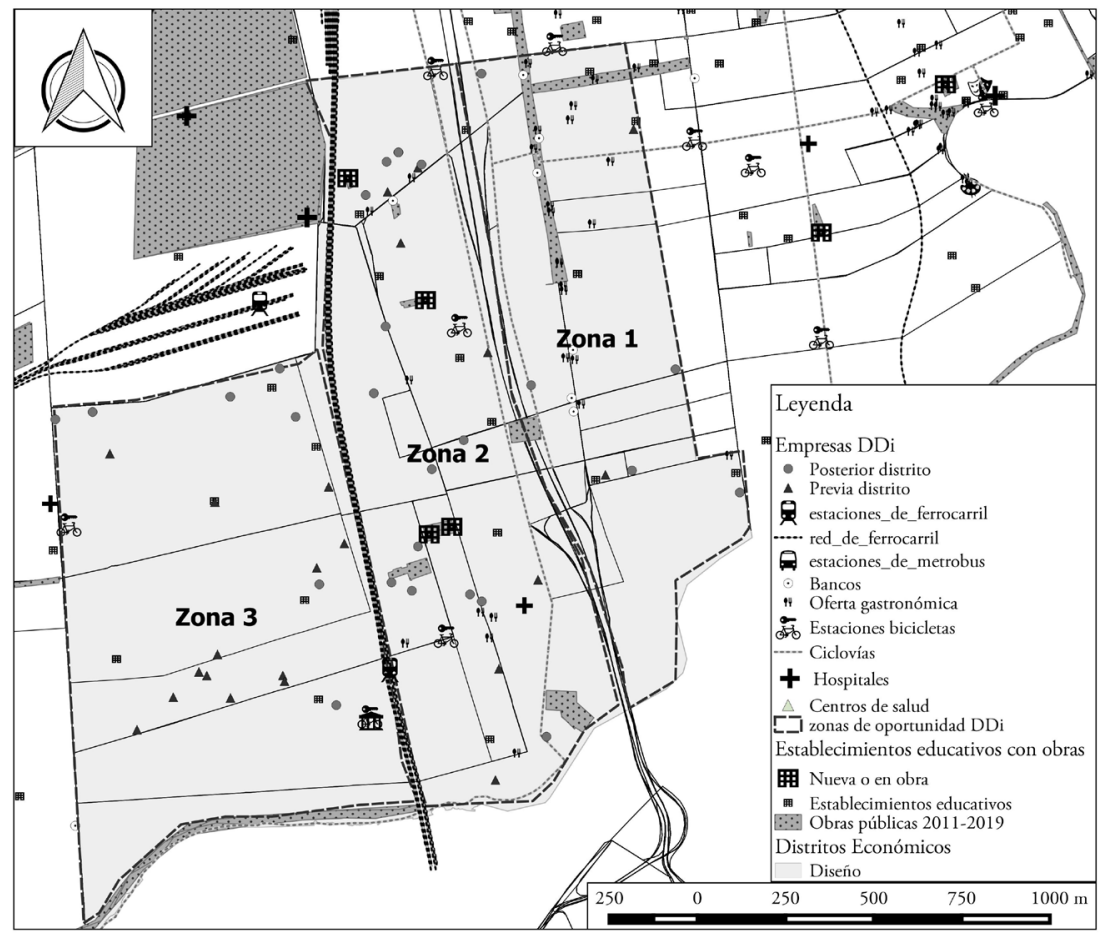

FUENTE: ELABORACIÓN PROPIA CON BASE EN INFORMACIÓN DEL GCBA

Al igual que en el DA, resuena la "apuesta" de inversión a futuro, debido a la lectura que hacen los empresarios sobre las características territoriales del área en cuestión. La accesibilidad, la cercanía al centro porteño, la disponibilidad de predios con usos industriales y, hasta entonces, con precios accesibles, convertían a este sector de Barracas en un área "con potencial" (coincidente con la lectura oficial). Estas características son valoradas positivamente por empresas de carácter industrial (no así por emprendimientos dedicados al diseño de indumentaria o publicitario, para quienes "el diseńo es Palermo" y no Barracas), incidiendo en su radicación aun antes de la creación del distrito. Por otro lado, si bien los incentivos fiscales son valorados positivamente, no tienen el mismo peso que en el DT; aquí solo representan "un 
ahorro", una especie de regalo que es bienvenido, pero que no incide demasiado en los respectivos negocios.

El Distrito del Diseńo, al igual que el de las Artes, produce un ajuste en relación con su modelo, el Distrito Tecnológico. En el DDi se incorpora un beneficio específico para los desarrolladores inmobiliarios -en este caso bajo la figura del "promotor de infraestructura para actividades de diseño"-, quienes pueden computar el 25\% de la inversión realizada a cuenta de ingresos brutos. Sin embargo, a pesar de este incentivo al desarrollo inmobiliario, hasta mediados de 2019 no había beneficiarios de este tipo en el distrito. Esto se debe, por un lado, a la ausencia de demanda (y, con ella, de financiamiento) para este tipo de emprendimientos, dado que no se mudaron muchas empresas. Esto se combina con la alta incidencia del suelo, que dificulta la rentabilidad de los proyectos, y con el tipo de desarrollos que requieren las actividades promovidas. Así lo explica el coordinador del DDi:

El tema es que la mayoría de los desarrolladores no quieren hacer galpones. Y la mayoría de las empresas tampoco se van a hacer un galpón de cero, cuando pueden hacer una puesta en valor del desarrollo que ya está. Entonces, tal vez lo que hacen es una remodelación de cáscara y chau (...). Entonces no es como el Tecnológico, que vos ves brutos desarrollos que se levantan y usan hermosas oficinas. (Entrevista al coordinador del DDi en el Ministerio de Economía y Finanzas, diciembre de 2018)

Sin embargo, a pesar de la ausencia de desarrollos vinculados al DDi, en el polígono comprendido por el distrito se observa un incremento en los precios promedio de venta de terrenos, departamentos y locales comerciales entre los años 2005 y 2017 (Lerena \& Gonzalez Redondo, 2019). El mayor incremento se dio con anterioridad a la creación del DDi, coincidiendo con la radicación del Distrito Tecnológico en el barrio lindero de Parque Patricios, por lo que es posible que este comportamiento de precios guarde relación con la expectativa generada a partir del DT. De todas formas, más allá de la influencia del DT, Barracas siempre ha tenido valores del suelo e inmobiliarios relativamente altos -comparado con otros barrios del sur de la ciudad-debido a su ubicación próxima al centro porteño, atributo valorado por los empresarios.

Retomando el análisis de las percepciones de los empresarios radicados en el DDi, advertimos que la mayoría no reconoce otros beneficios o actividades significativas vinculadas al distrito, más allá de la exención en II. BB. De los relatos tampoco se advierte una identidad común al distrito, a excepción de algunas referencias al Centro Metropolitano del Diseño (CMD) como espacio articulador y como "semilla" del DDi. Los empresarios consultados tampoco tienen en claro cuántas empresas radicadas hay, ni a qué se dedican. A diferencia de lo que sucede en los otros distritos, no se advierte ningún tipo de vínculos entre las firmas instaladas, ni siquiera entre aquellas del mismo rubro:

Mi sensación es que todos nos entusiasmamos para tener un beneficio y murió ahí. Quedó ahí. Estoy tranquilo, que tengo los beneficios, tengo ingresos brutos. Y como que no hubo más entusiasmo o más empuje de desarrollar otras cosas. (Entrevista a Leonel, empresario DDi, mayo de 2019) 
De todas formas, algunos de los entrevistados reconocen algunos esfuerzos del gobierno local orientados a articular a las empresas entre sí, sobre todo en los orígenes del distrito. Destacan algunos desayunos y encuentros en el CMD que, en general, sirvieron para presentar demandas comunes y problemáticas particulares al Estado. También recuerdan invitaciones a capacitaciones y eventos puntuales, a los que no asistieron por falta de tiempo o por no sentirse interpelados. De este modo, los esfuerzos públicos orientados a fomentar el denominado "efecto clúster" (Porter, 1998) son percibidos como actos de "buena voluntad" que resultan insuficientes, sobre todo desde 2017, momento en que los distritos perdieron peso en la agenda pública local (Gonzalez Redondo, 2020b). Si bien hay intenciones de armar un consorcio similar a los que se generaron en los otros distritos, la gran diversidad de actividades promovidas y la delicada situación económica que atraviesan muchas de estas empresas atentan contra esta iniciativa:

A diferencia de otros distritos, en este distrito la mayoría de las empresas, honestamente hablando, salvo algunas, están con la atención puesta en sobrevivir. Sobrevivir en la diaria, digamos, porque entre los costos y la poca venta están muy ahorcadas. Este año fue muy difícil para el sector. (...) Entonces, a la hora de proponerles hacer acciones, la realidad es que las empresas están muy golpeadas y te manifiestan cierta bronca. (Entrevista al coordinador del DDi en el Ministerio de Economía y Finanzas, diciembre de 2018)

Los intentos del gobierno local encuentran sus limitaciones cuando se enfrentan a situaciones macroeconómicas que los exceden, dado que las interpretaciones que los empresarios hacen sobre la coyuntura política y económica inciden en el modo en que organizan su acción (Arqueros, 2017; Beltrán, 2007; Gaggero, 2012) y, así, moldean el derrotero de la política local. De hecho, para los empresarios entrevistados el contexto económico es uno de los factores que va en detrimento de la articulación entre los actores del distrito.

De este modo, la propuesta del gobierno local, que -siguiendo la premisa del clúster- planteaba primero radicar las empresas; luego, fomentar los vínculos entre sí para que, finalmente, "el gobierno de la ciudad pueda correrse y dejar paso a que todo ese ecosistema, esa dinámica, se desenvuelva”, ${ }^{6}$ parece haber quedado en el primer paso. Entre los factores que dificultan la atracción de inversiones, advertimos aquellos que los funcionarios reconocieron al momento de diseñar la política: dificultades que se relacionan con la ley misma, con las particularidades de las actividades promovidas y con las características de Barracas. Todo ello agravado por la coyuntura económica, que golpea a muchas de las industrias promocionadas.

\section{Reflexiones finales}

El análisis de las percepciones y estrategias de los actores privados involucrados en cada distrito permite discutir algunos de los supuestos en los que se apoya esta política. Primero, que la concentración geográfica de empresas genera "sinergias" que 
redundan en procesos innovadores y de crecimiento económico. Por lo contrario, vemos que las relaciones entre los actores empresariales son complejas, primando dinámicas de competencia y asilamiento entre actores con intereses, interpretaciones y prioridades divergentes. A pesar de los esfuerzos públicos -diferenciales en cada caso- para promover el "efecto clúster" (Porter, 1998), los actores empresariales tuvieron dificultades para sostener una acción colectiva organizada (Dossi \& Lissin, 2011) a lo largo del tiempo. Especialmente en el Distrito del Diseńo, la interpretación que los actores hacen de la coyuntura incide en sus decisiones y obtura la generación del "efecto clúster". Así, el impacto que tiene el contexto nacional permite cuestionar la autonomía de los gobiernos locales frente a coyunturas que los exceden. De conjunto, estos análisis invitan a discutir la noción de relaciones armónicas y libres de conflicto entre los distintos actores que integran el clúster. Esta idea encuentra sus limitaciones al reconocerse en el territorio la existencia de intereses, expectativas y definiciones éticas, estéticas y políticas divergentes, que hacen que la articulación no sea una panacea, ni un objetivo al que se pueda aspirar.

Otro supuesto que se pone en cuestión es aquel que confía en las "sinergias público-privadas" y en los procesos de gobernanza urbana para propiciar el desarrollo de "áreas deterioradas". A pesar de los esfuerzos públicos por crear el "clima de negocios" y atraer inversiones a los distritos, la respuesta del sector privado tiende a ser limitada en su acompañamiento a la temporalidad y la ubicación de las intervenciones públicas. Si bien prima un discurso que sostiene que el Estado "no pone un peso" (Gonzalez Redondo, 2020b) y que el privado "apuesta" a los distritos, advertimos que el sector privado se limita en general a aprovechar los incentivos públicos asumiendo muy pocos riesgos, mostrando con ello los límites de la gobernanza urbana.

Asimismo, el Estado facilitador y mediador no favorece con la misma intensidad a todos los actores ni se despliega homogéneamente en el territorio, generando con ello condiciones propicias para una respuesta heterogénea de los actores privados. En conjunto, estas intervenciones públicas y privadas originaron procesos territoriales desiguales entre e intra distritos, reforzando las desigualdades socioterritoriales preexistentes y persistentes. En el Distrito Tecnológico, el acceso en propiedad de las empresas TIC garantizó el capital necesario para la emergencia de un nuevo mercado de oficinas, que impactó el sistema de usos del suelo y la estructura de rentas en el área. En el Distrito del Diseño también se advierte una valorización inmobiliaria y del suelo, que es previa a la creación del distrito y se asocia a las expectativas generadas por su modelo vecino. En el Distrito de las Artes, en cambio, a pesar de los esfuerzos públicos y privados, todavía no se ha generado un cambio en el sistema de usos del suelo que derive en la estructura de rentas de la zona (Jaramillo, 2009). Sin embargo, ello no impidió que se produjera un impacto negativo en los sectores más vulnerables del barrio La Boca.

El análisis comparado de los tres distritos da cuenta de la heterogeneidad en los resultados de una misma política, confirmando la importancia de un análisis situado de aquellas políticas que circulan globalmente - muchas veces como "recetas" posibles de aplicar, pero que se desenvuelven localmente-, a partir de las fricciones que impone cada territorio. Asimismo, estos hallazgos permiten cuestionar los 
beneficios de la articulación público-privada en la gestión de la ciudad, advirtiendo que se producen ganadores (algunos actores empresariales) y perdedores (las arcas públicas) en esta articulación. Parafraseando a Vainer (2000), se configura la ciudad como mercancía, como objeto para ser vendido y consumido; aunque la ciudad como empresa se queda a medio camino, solo retoma de la última sus estrategias y argumentos de venta, pero no su capacidad para recuperar la inversión, diferenciándose del empresarialismo urbano implementado en ciudades del norte global (Harvey, 1989; Leitner, 2002). En este sentido, el abordaje desde la sociología económica constituye un aporte al campo de los estudios urbanos para pensar las relaciones entre actores estatales y empresariales en la producción de ciudad. Este abordaje brinda herramientas para el análisis de los arreglos entre actores públicos y privados en un nivel más sustantivo y, de esta forma, permite dotar de contenido a algunas categorías más abstractas, como la de "neoliberalismo realmente existente" (Theodore et al., 2009).

\section{Referencias bibliográficas}

Aguilar, L. (2006). Gobernanza y gestión pública. Fondo de Cultura Económica.

Amossy, R. (2000). Le plausible et l'évident: doxa, interdiscours, topiques. En L'argumentation dans le discours. Discours politique, littérature d'idées, fiction (pp. 99-126). Nathan Université.

Arqueros, S. (2017). La política de desarrollo de la Comuna 8 de la ciudad de Buenos Aires (1996-2015). Quid 16, 11, 331-337. https://publicaciones.sociales.uba.ar/index.php/ quid16/article/view/3608/pdf

Banco Mundial (вм). (2009). Una nueva geografia económica. Informe sobre el Desarrollo, $I(1), \quad$ 1-48. http://siteresources.worldbank.org/INTWDR2009/Resources/WDR_ OVERVIEW_ES_Web.pdf

Beltrán, G. (2007). La acción empresarial en el contexto de las reformas estructurales de las décadas de los ochenta y noventa en Argentina. Tesis de doctorado en Ciencias Sociales, Universidad de Buenos Aires, Argentina.

Beltrán, G. (2011). Escenas de la vida corporativa. Conocimiento y toma de decisión en las organizaciones. Apuntes de Investigación del cecyp, 14(19), 103-130. https:// publicaciones.sociales.uba.ar/index.php/apuntescecyp/article/view/4096

Castellani, A. (2006). La relación entre intervención estatal y comportamiento empresario. Herramientas conceptuales para pensar las restricciones al desarrollo en el caso argentino. Primera Jornada de Estudios Sociales de la Economía. Instituto de Altos Estudios Sociales, Universidad Nacional de General San Martín, julio de 2006. https:// $\mathrm{xdoc} . \mathrm{mx} /$ documents/la-relacion-entre-intervencion-estatal-y-comportamientoempresario-5e309694ad183

Castellani, A. \& Borrastero, C. (2011). ¿Estrategia o privilegios? Estado y empresarios en el desarrollo del sector software y servicios informáticos de la ciudad de Córdoba (20002010). IX Jornadas de Sociología. Facultad de Ciencias Sociales, Universidad de Buenos Aires, 2011. https://www.aacademica.org/000-034/422 
Castellani, A. \& Gaggero, A. (2012). La retirada heterogénea: estrategias y desempeños de los grupos económicos nacionales en la Argentina de la década de 1990. Apuntes, 39(70), 203-238. https://doi.org/10.21678/apuntes.70.650

Comisión Económica para América Latina y el Caribe (CEPAL). (2018). Plan de Acción Regional para la implementación de la Nueva Agenda Urbana en América Latina y el Caribe (2016-2036). CEPAL. https://repositorio.cepal.org/bitstream/handle/11362/42144/2/ S1800033_es.pdf

Dossi, M. \& Lissin, L. (2011). La acción empresarial organizada: Propuesta de abordaje para el estudio del empresariado. Revista Mexicana de Sociología, 73(3), 415-443. http://www. jstor.org/stable/23075714

Gaggero, A. (2012). Estado, mercado y dinámicas organizacionales. Un abordaje de las estrategias empresarias a través de la sociología económica. Papeles de Trabajo, 6(9), 3757. https://revistasacademicas.unsam.edu.ar/index.php/papdetrab/article/view/249

Goicoechea, M. E. (2016). Distritos creativos en el sur de la Ciudad de Buenos Aires (20082015). Renovación urbana y nuevas lógicas de segregación. Quid 16, 9, 224-227. https://publicaciones.sociales.uba.ar/index.php/quid16/article/view/2897/pdf_14

Gonzalez Redondo, C. (2018). Los modelos de aglomeración realmente existentes. Ciudades, 29(120), 34-43.

Gonzalez Redondo, C. (2019). La política de distritos en la ciudad de Buenos Aires. Una exploración sobre los roles que asume el Estado local en los procesos de renovación urbana. Seminario El Complejo Urbano Financiero-Inmobiliario en América Latina. Santiago de Chile, 4 y 5 de abril de 2019.

Gonzalez Redondo, C. (2020a). El vaciamiento que antecede la renovación. Reflexiones a partir del proyecto del distrito gubernamental en el sur de la ciudad de Buenos Aires. Territorios, (42), 1-22. http://dx.doi.org/10.12804/revistas.urosario.edu.co/ territorios/a.7228

Gonzalez Redondo, C. (2020b). Gobernanza urbana: reflexiones a partir de los distritos económicos de la ciudad de Buenos Aires. Revista INVI, 35(100), 91-114. http://dx.doi. org/4 0 6 7/S0718-83582020000300091

Gonzalez Redondo, C. (2020c). De modelos internacionales y adaptaciones locales. El proceso de movilidad de la política de distritos económicos de la ciudad de Buenos Aires (20082019). Revista de Geografia Norte Grande, (77), 91-108. https://dx.doi.org/10.4067/ S0718-34022020000300091

Guevara, T. (2010). Políticas habitacionales y procesos de producción del hábitat en la Ciudad de Buenos Aires. El caso de La Boca. Cuaderno Urbano. Espacio, cultura, sociedad, 11(11), 81-99. https://www.redalyc.org/pdf/3692/369236772004.pdf

Harvey, D. (1989). From managerialism to entrepreneurialism: the transformation in urban governance in late capitalism. Geografiska Annaler, 71(1), 3-17. https://doi. org $/ 10.2307 / 490503$

Harvey, D. (2005). Breve historia del neoliberalismo. Akal.

Herzer, H. (Comp.). (2008). Con el corazón mirando al sur. Espacio.

Jaramillo, S. (2009). Hacia una teoría de la renta del suelo urbano. Universidad de los Andes, Bogotá.

Leitner, H. (2002). Cities in pursuit of economic growth. Political Geography Quarterly, 9(2), 146-170. https://doi.org/10.1016/0260-9827(90)90016-4 
Lerena, N. (2019). Políticas de renovación urbana y valorización del mercado inmobiliario y de suelo, en el sur de la Ciudad de Buenos Aires. El caso del Distrito Tecnológico. 2008-2018. Tesis para la obtención del grado de Doctor en Geografía de la Facultad de Filosofía y Letras de la Universidad de Buenos Aires, Argentina. http://repositorio.filo.uba.ar/bitstream/ handle/filodigital/11457/uba_ffyl_t_2019_se_lerena.pdf?sequence=1\&isAllowed=y

Lerena, N. \& Gonzalez Redondo, C. (2019). Políticas de renovación urbana y valorización del mercado inmobiliario y de suelo. Los distritos económicos en la Ciudad de Buenos Aires. Revista de Geografia Norte Grande, (78), 163-192. http://dx.doi.org/10.4067/ S0718-34022021000100163

Marques, E. (2013). Government, political actors and governance in urban policies in Brazil and São Paulo: concepts for a future research agenda. Brazilian Political Science Review, 7(3), 8-35. https://doi.org/10.1590/s1981-38212013000300001

Naciones Unidas (onU). (2017). Nueva Agenda Urbana. Hábitat iii. https://uploads.habitat3. org/hb3/NUA-Spanish.pdf

Peck, J. \& Theodore, N. (2010). Mobilizing policy: Models, methods, and mutations. Geoforum, 41(2), 169-174. https://doi.org/10.1016/j.geoforum.2010.01.002

Peet, R. (2001). Globalización y neoliberalismo. En La maldita trinidad (Cap. 1). Laetoli.

Porter, M. (1995). The competitive advantage of the inner city. Harvard Bussiness Review, 73(3), 55-71. https://www.academia.edu/2917825/The_competitive_advantage_of_ the_inner_city

Porter, M. (1998). Clusters and the new economic competition. Harvard Business Review, 76(6), 77-90.

Rodríguez, M. C. \& Di Virgilio, M. (2014). Ciudad de Buenos Aires: políticas urbanas neoliberales, transformaciones socio-territoriales y hábitat popular. Revista de Direito da Cidade, 6(2), 323-347. https://doi.org/10.12957/rdc.2016.19115

Socoloff, I. (2013). Reflexiones en torno a las relaciones entre empresa, estado y ciudad: Un estudio a partir del caso irsa en Buenos Aires (1991-2012). Tesis de doctorado en Ciencias Sociales, Universidad de Buenos Aires, Argentina.

Socoloff, I. (2018). Grandes desarrolladores inmobiliarios: hacia una tipología de sus estrategias de inversión y financiamiento entre 2002 y 2015. Jornadas "Empresas, Empresarios y Burocracias Estatales en la Producción del Espacio Urbano a través de la Historia".

Theodore, N., Peck, J. \& Brenner, N. (2009). Urbanismo neoliberal: la ciudad y el imperio de los mercados. Temas Sociales SUR, (66). http://www.sitiosur.cl/r.php?id=898

Vainer, C. (2000). Pátria, empresa e mercadoria. Notas sobre a estratégia discursiva do Planejamento Estratégico Urbano. En O. Arantes, C. Vainer \& E. Maricato, A cidade do pensamento único (pp. 75-102). Editora Vozes.

Zurbriggen, C. (2011). Gobernanza: una mirada desde América Latina. Perfiles Latinoamericanos, 19(38), 39-64. http://www.scielo.org.mx/scielo.php?script=sci_arttext\&pid=S0188$76532011000200002 \& \operatorname{lng}=e s \& n r m=i s o \& t \operatorname{lng}=e s$ 